\title{
Efeito terapêutico do "cross-linking" corneano na ceratopatia bolhosa sintomática
}

\author{
Therapeutic effect of corneal cross-linking on symptomatic \\ bullous keratophaty
}

\author{
Diego Nery Benevides Gadelha ${ }^{1}$ \\ Bernardo Menelau Cavalcanti ${ }^{2}$ \\ Vasco Bravo Filho ${ }^{3}$ \\ Newton Andrade Júnior ${ }^{4}$ \\ Neylane Nyéria Coelho Batista ${ }^{5}$ \\ Ana Cecília de Sousa Leão Escarião ${ }^{6}$ \\ Roberta Ventura Urbano ${ }^{7}$
}

Trabalho realizado na Fundação Altino Ventura (FAV) - Recife (PE) - Brasil.

${ }^{1}$ Médico, Aluno do segundo ano do Curso de Especialização em Oftalmologia da Fundação Altino Ventura (FAV) - Recife (PE) - Brasil.

${ }^{2}$ Médico, Aluno do segundo ano do Curso de Especialização em Oftalmologia da FAV - Recife (PE) - Brasil. Médico, Aluno do segundo ano do Curso de Especialização em Oftalmologia da FAV - Recife (PE) - Brasil.

${ }^{4}$ Médico, Aluno do segundo ano do Curso de Especialização em Oftalmologia da FAV - Recife (PE) - Brasil.

${ }^{5}$ Médica, Aluna do primeiro ano do Curso de Especialização em Oftalmologia do Hospital das Clínicas da Universidade Federal de Pernambuco (UFPE) - Recife (PE) - Brasil.

Médica, Staff do Departamento de Córnea e Doenças Externas da FAV e Hospital de Olhos de Pernambuco (HOPE) - Recife (PE) - Brasil.

7 Diploma em Saúde Comunitária Ocular pela London School of Hygiene and Tropical Medicine.

Endereço para correspondência: Fundação Altino Ventura (FAV). Rua da Soledade, 170 - Recife (PE)

CEP 50070-040

E-mail: fav@fundacaoaltinoventura.org.br

Recebido para publicação em 19.01.2009

Aprovação em 07.06.2009

Nota Editorial: Depois de concluída a análise do artigo sob sigilo editorial e com a anuência da Dra. Myrna Serapião dos Santos sobre a divulgação de seu nome como revisora, agradecemos sua participação neste processo.

\begin{tabular}{l} 
RESUMO \\
\hline Objetivo: Avaliar o efeito do "cross-linking” corneano na ceratopatia \\
bolhosa sintomática e seu impacto na acuidade visual, espessura corneana \\
e sintomatologia dolorosa. Métodos: Doze pacientes com ceratopatia \\
bolhosa sintomática foram incluídos. Exame clínico com questionário \\
específico para a pesquisa com escala de dor (escala visual analógica \\
numérica), acuidade visual e mensuração da espessura corneana foi \\
realizada pré "cross-linking" corneano, 7,30 e 60 dias após. Em todos \\
os pacientes o tratamento com UVA-crosslinking foi realizado após \\
abrasão do epitélio corneano na lâmpada de fenda e instilação de \\
solução de riboflavina 0,1\% a cada 5 minutos por 30 minutos. Após esse \\
período o paciente foi submetido à exposição à luz ultravioleta A \\
(UVA), utilizando riboflavina e anestesia tópica a cada 5 minutos por \\
30 minutos. O paciente utilizou colírios de ofloxacina $0,3 \%$ e lágrima \\
artificial até completa reepitelização. O teste de Friedman foi usado para \\
comparar as médias das frequências da acuidade visual, sintomatologia \\
dolorosa e espessura central da córnea. Os valores de "p" menor que \\
0,05 foram considerados como estatisticamente significantes. Resul- \\
tados: Doze olhos de 12 pacientes com erosões epiteliais recorrentes \\
foram tratados. O tempo de seguimento foi de dois meses. Foi obser- \\
vada redução significante da dor (p<0,001). As medidas da espessura \\
corneana e da acuidade visual não sofreram alterações estatisticamente \\
significantes. Conclusão: Foi constatado o potencial de aplicação do \\
"cross-linking” corneano no tratamento de pacientes com dor causada \\
por ceratopatia bolhosa. É necessário maior seguimento para confirmar \\
se os resultados podem ser reproduzidos em longo prazo. \\
\hline
\end{tabular}

Descritores: Córnea; Topografia da córnea; Reagentes para ligações cruzadas; Transplante de córnea; Dor; Agentes fotossensibilizantes/uso terapêutico; Riboflavina/uso terapêutico; Raios ultravioleta; Acuidade visual

\section{INTRODUÇ̃̃̃O}

A transparência da córnea é mantida pela bomba iônica no seu endotélio, responsável pela deturgescência e desidratação estromal ${ }^{(1)}$. A ceratopatia bolhosa (CB) é uma desordem causada pela falha desse mecanismo, resultando em edema estromal e, invariavelmente, aparecimento de bolhas epiteliais ou subepiteliais ${ }^{(1-4)}$. As causas mais comuns de CB são os procedimentos cirúrgicos intraoculares incluindo facectomia e ceratoplastia penetrante, distrofia endotelial de Fuchs, trauma e glaucoma refratário ${ }^{(1-4)}$. A CB produz, frequentemente, sintomas, como diminuição da acuidade visual, dor, fotofobia e epífora ${ }^{(2)}$. 
Transplante de córnea é o método mais efetivo para alívio dos sintomas e recuperação visual ${ }^{(2-3)}$. Quando este tratamento é inviável do ponto de vista clínico ou não está disponível, métodos conservadores podem ser usados. Dor ocular e defeitos epiteliais podem ser tratados com administração tópica de anti-inflamatórios, lubrificantes e soluções hipertônicas ${ }^{(5-6)}$, além do uso de lente de contato terapêutica ${ }^{(7)}$. Alternativamente, vários tratamentos cirúrgicos têm sido utilizados na $\mathrm{CB}$, incluindo punção do estroma anterior, criopexia (cauterização), recobrimento conjuntival, transplante de membrana amniótica, ceratectomia fototerapêutica por excimer laser (PTK) e ceratoplastia lamelar posterior ${ }^{(3)}$.

O "cross-linking" do colágeno corneano com a riboflavina (vitamina B2) é uma técnica inovadora com o objetivo de enrijecer o tecido corneano ${ }^{(8-9)}$. A luz ultravioleta A (UVA) associada à riboflavina cria novas ligações entre as moléculas de colágenos adjacentes, produzindo aumento da espessura da córnea bem como diminuindo sua maleabilidade ${ }^{(10)}$. A irradiação por meio da UVA faz com que ocorra perda do equilíbrio interno das moléculas de riboflavina, que atua como um fotomediador, criando radicais livres que induzem novas ligações entre fibrilas de colágeno ${ }^{(11)}$. Esta reestruturação das fibras de colágeno ocasiona aumento da força biomecânica da córnea ${ }^{(10-11)}$.

A indicação clínica do "cross-linking" corneano está limitada aos casos de "melting" corneano, degeneração marginal pelúcida, ceratocone e ectasias iatrogênicas após cirurgias refrativas com laser in situ keratomileusis (LASIK) ${ }^{(12-13)}$. Publicações que tenham analisado os efeitos terapêuticos do "cross-linking" corneano na ceratopatia bolhosa são escassas e metodologicamente incompletas.

O objetivo do estudo é analisar o efeito terapêutico do "cross-linking" corneano induzido por UVA/riboflavina na ceratopatia bolhosa sintomática, demonstrando a viabilidade deste tratamento e sua influência na acuidade visual, espessura corneana e sintomatologia dolorosa.

\section{MÉTODOS}

Foram incluídos no estudo 12 portadores de CB sintomática por um período igual ou maior que 12 meses, resistente ao tratamento clínico conservador, que não possuíam indicação para transplante penetrante de córnea, ou estivessem na fila de espera para a realização do transplante.

Foram excluídos todos os pacientes que possuíam espessura central da córnea menor que 400 micra; antecedente de herpes ocular ou ao redor dos olhos; portador de outras doenças ou cicatrizes corneanas; antecedente de displasia corneana; lesões tumorais conjuntivais; período de gravidez ou amamentação; antecedente de má cicatrização corneana e alergia à riboflavina.

Os pacientes foram avaliados, previamente ao tratamento e no $7^{\circ}, 30^{\circ}$ e $60^{\circ}$ dias após o tratamento, com questionário estruturado para o estudo, avaliando sintomatologia e dor através da escala visual analógica numérica $(\mathrm{EVA})^{(14)}$. Além do questionário, foi realizada avaliação oftalmológica completa, que incluiu: acuidade visual de alto contraste nãocorrigida (AVL), utilizando a tabela ETDRS ${ }^{\mathrm{TM}}$ (Lighthouse Inc., Nova Iorque, EUA); biomicroscopia detalhada, na qual foi observada a superfície da córnea com e sem instilação de fluoresceína, vascularização superficial e profunda; representação gráfica detalhada do quadro; paquimetria ultrassônica (Ultrasonic Pachymeter Model 850, Humphrey Instruments, Inc).

Abrasão do epitélio da córnea medindo $9 \mathrm{~mm}$ de diâmetro foi realizada com lâmina de bisturi número 11 , gotas da solução de riboflavina $0,1 \%$ foram instiladas a cada 5 minutos intercaladas com colírio anestésico, em um período de 30 minutos antes do procedimento e durante a aplicação da radiação. A córnea foi exposta a luz UVA com comprimento de onda de $370 \pm 5 \mathrm{~nm}$ e uma irradiância de $3 \mathrm{~mW} / \mathrm{cm}^{2}$ por um período total de 30 minutos, correspondendo a uma dose total de 3,4 J ou a uma exposição de radiação de $5,4 \mathrm{~J} / \mathrm{cm}^{2}$ de córnea. Foi utilizado para esta exposição o aparelho X-Link (Corneal Crosslink System, Opto Global Pty Ltd). Ao final, colocada uma lente de contato terapêutica e prescrito colírio antibiótico (ofloxacino 0,3\%) até recuperação epitelial.

Para a avaliação da evolução das medidas EVA, AVL e paquimetria nos períodos pré, 7, 30 e 60 dias após o tratamento, foi aplicado o teste não paramétrico de Friedman ${ }^{(15)}$ que é aplicado no caso de medidas pareadas com mais de dois períodos de avaliação. No caso onde houve significância estatística, foi aplicado o teste de comparações múltiplas com a finalidade de determinar onde se encontra a diferença estatística. Optou-se por utilizar metodologia não paramétrica devido ao número pequeno de observações.

Foram considerados estatisticamente significantes os resultados cujos níveis descritivos (valores de $\mathrm{p}$ ) foram inferiores a 0,05 . O software utilizado na execução dos cálculos estatísticos foi o SPSS versão 12.0.

O estudo foi aprovado pelo Comitê de Ética em Pesquisa da Fundação Altino Ventura. Todos os indivíduos que participaram do estudo foram esclarecidos quanto à investigação e assinaram o Termo de Consentimento Livre e Esclarecido.

\section{RESULTADOS}

Dos 12 pacientes incluídos no estudo, $6(50,0 \%)$ eram do sexo masculino e $6(50,0 \%)$ do feminino. A idade média foi de 66 anos (variando entre 49 e 85 anos). As principais causas de ceratopatia bolhosa foram: pós-facectomia (5 pacientes), trauma (2 pacientes), falência secundária de transplante de córnea (1 paciente), falência secundária de transplante de córnea em olho pseudofácico ( 2 pacientes), distrofia endotelial de Fuchs (2 pacientes).

Nenhum caso de infecção corneana ou inflamação intraocular foi relatado. Apenas uma paciente apresentou retardo no tempo de reepitelização (15 dias). 
A sintomatologia dolorosa foi avaliada por meio da escala visual analógica numérica (EVA), onde zero significa ausência total de dor e 10 o nível de dor máxima suportável pelo paciente - tabela 1.

Através dos resultados obtidos acima, pode-se afirmar que existe uma redução significativa $(\mathrm{p}<0,05)$ dos valores da EVA do período pré em relação aos momentos pós-tratamento, já verificando uma melhora após 7 dias. Depois do tratamento não existe diferença estatisticamente significante $(p>0,05)$ entre os momentos avaliados (7,30 e 60 dias) - tabela 2. Esses resultados podem ser melhor visualizados no gráfico 1 .

Analisando a paquimetria, houve uma tendência discreta para a diminuição da espessura corneana central, entretanto, pode-se afirmar que para a amostra estudada não existe uma diferença estatisticamente significante dos valores entre os períodos pré, 30 e 60 dias após o tratamento $(\mathrm{p}>0,05)$ - tabela 3 .

Através dos resultados obtidos na análise da acuidade visual de alto contraste não corrigida (AVL), pode-se afirmar que para a amostra estudada não existe diferença estatisticamente significante dos valores da AVL entre os períodos pré, 7,30 e 60 dias após o tratamento $(p>0,05)$ - tabela 4.

\section{DISCUSS ÃO}

A ceratopatia bolhosa é causada pela falha da capacidade do endotélio corneano em manter o estado fisiológico de desidratação e transparência da córnea ${ }^{(16)}$. Devido à estrutura mecânica das fibras corneanas, uma quebra na integridade da barreira epitelial resulta em um modesto aumento da espessura corneana: valores acima de 0,6 mm são raros. Em contrapartida, descompensação endotelial é seguida de abundante hidratação estromal, com espessura entre $0,8-1,0 \mathrm{~mm}^{(17)}$. O ede-

Tabela 1. Resultado da análise da evolução da EVA no período de avaliação

\begin{tabular}{|lcccccc|} 
EVA & N & Média & $\begin{array}{c}\text { Mediana } \\
\text { Pré }\end{array}$ & $\begin{array}{l}\text { Desvio } \\
\text { padrão }\end{array}$ & Mínimo & Máximo \\
7 dias & 12 & 8,58 & 9,00 & 1,51 & 7 & 10 \\
30 dias & 12 & 3,75 & 2,00 & 3,14 & 0 & 10 \\
60 dias & 12 & 4,50 & 4,00 & 3,18 & 1 & 10 \\
EVA= escala visual analógica numérica; p-valor < 0,001 & & 10 \\
\hline
\end{tabular}

Tabela 2. Resultado das comparações múltiplas para EVA

$\begin{array}{lr}\text { Comparações - EVA } & \text { p-valor } \\ \text { Pré x } 7 \text { dias } & 0,001^{*} \\ \text { Pré x } 30 \text { dias } & 0,001^{*} \\ \text { Pré x } 60 \text { dias } & 0,001^{*} \\ 7 \text { dias x } 30 \text { dias } & 0,267 \\ 7 \text { dias x } 60 \text { dias } & 0,455 \\ 30 \text { dias x } 60 \text { dias } & 0,491 \\ \text { EVA= escala visual analógica numérica; * }{ }^{*}=\text { estatisticamente significante }\end{array}$

ma estromal e a ruptura de bolhas epiteliais e subepiteliais irritam as terminações nervosas corneanas, produzindo dor e desconforto na superfície ocular ${ }^{(18)}$.

Todos os pacientes do estudo apresentavam dor de importante intensidade antes da aplicação de UVA/riboflavina, com média na escala visual analógica numérica de $8,58( \pm 1,51)$. Após a realização do procedimento observou-se uma considerável redução da dor já na primeira semana, com manutenção estatisticamente significante em 30 e 60 dias.

O tratamento afeta a inervação corneana. A desepitelização mecânica remove as fibras nervosas intraepiteliais e causa dano às fibras subepiteliais ${ }^{(18)}$. $\mathrm{O}$ efeito sinérgico entre abrasão mecânica e exposição à UVA/riboflavina (induzindo fotonecrose e apoptose) promovem perda de fibras nervosas estromais anteriores e subepiteliais ${ }^{(18)}$. A combinação desses efeitos com o aumento da rigidez corneana induzido pelo "cross-linking" são provavelmente responsáveis pela melhora da dor nos pacientes da amostra.

Em relação à análise da paquimetria não foi observada alteração significante na espessura corneana no período estudado. Estudos prévios demonstraram um decréscimo maior que $10 \%$ na espessura da córnea, isso pode ser explicado pelas mudanças arquitetônicas nas fibrilas de colágeno geradas pela exposição à UVA/riboflavina, que aumenta as pontes entre fibrilas e torna mais difícil o influxo de líquido no estroma ${ }^{(19)}$. Outra possibilidade seria de que as diferenças entre as séries resultassem apenas de bases de tamanho amostral.

A melhora da acuidade visual nos pacientes com CB está intimamente relacionada à redução do edema e aumento da transparência corneana. Como não houve alterações nessas



Gráfico 1 - Box-Plot dos valores da escala visual analógica numérica (EVA) para os períodos pré, 7, 30 e 60 dias após o tratamento 


\begin{tabular}{|lcccccc|}
\hline & \multicolumn{7}{c|}{ Tabela 3. Resultado da análise da evolução da paquimetria no período de avaliação } \\
Paquimetria $(\mu \mathrm{m})$ & $\mathbf{N}$ & Média & Mediana & Desvio padrão & Mínimo & Máximo \\
Pré & 12 & 803,00 & 826,00 & 137,97 & 561 \\
30 dias & 12 & 735,50 & 708,50 & 149,15 & 544 & 978 \\
60 dias & 12 & 737,75 & 727,50 & 133,56 & 550 \\
p-valor $=0,338$ & \multicolumn{7}{c}{} \\
\hline
\end{tabular}

\begin{tabular}{|c|c|c|c|c|c|c|}
\hline AVL & $\mathbf{N}$ & Média & Mediana & Desvio padrão & Mínimo & Máximo \\
\hline Pré & 12 & 0,022 & 0,010 & 0,037 & 0,00 & 0,13 \\
\hline 7 dias & 12 & 0,024 & 0,010 & 0,036 & 0,00 & 0,10 \\
\hline 30 dias & 12 & 0,024 & 0,015 & 0,036 & 0,00 & 0,13 \\
\hline 60 dias & 12 & 0,024 & 0,015 & 0,036 & 0,00 & 0,13 \\
\hline
\end{tabular}

variáveis é esperado que não haja incremento de linhas de visão nesses pacientes.

Outros tratamentos utilizados para $\mathrm{CB}$ como punção de estroma anterior, recobrimento conjuntival e transplante de membrana amniótica podem levar ao decréscimo da acuidade visual. A lente de contato terapêutica mostra-se eficaz, porém, além da necessidade de trocas frequentes, está associada ao aumento da vascularização e do risco de infecção quando utilizada por tempo prolongado ${ }^{(2)}$.

Apenas um paciente apresentou retardo no tempo de reepitelização corneana. Nos demais nenhum efeito adverso relacionado ao procedimento ou ao período de recuperação foram relatados. É necessário um tempo maior de seguimento para confirmar se esses resultados podem ser reproduzidos em longo prazo.

\section{CONCLUSÃO}

A indução de "cross-linking" corneano através da aplicação de UVA/riboflavina mostrou-se eficaz no controle da sintomatologia dolorosa da ceratopatia bolhosa. Apresentou ainda manutenção da espessura corneana e da acuidade visual. Um tempo maior de seguimento é necessário para avaliar se os resultados são reprodutíveis em longo prazo.

\section{ABSTRACT}

Purpose: To evaluate the corneal cross-linking (CXL) effect on symptomatic bullous keratopathy, and its impact on the visual acuity, corneal thickness and pain symptomatology. Methods: Twelve patients with symptomatic bullous keratophaty were included. A clinical examination with a specific questionnaire (pain scale - visual numeric analog scale), visual acuity, and corneal thickness measures was performed before corneal cross-linking and 7, 30 and 60 days after the procedure. In all patients, the treatment with the UVA-crosslinking was done after corneal abrasion on the slit-lamp and instillation of $0.1 \%$ riboflavin in saline for every five minutes, lasting 30 minutes. Subsequently, the patient underwent the UVA light exposure, using riboflavin and topical anesthesia, every five minutes lasting 30 minutes. The patient received $0.3 \%$ ofloxacine drops, and artificial tears until complete reepithelization. The Friedman test was used to compare the means of frequencies of visual acuity, pain symptoms and corneal thickness. A "p" value $<0.05$ was considered for rejecting the null hypothesis. Results: Twelve eyes from 12 patients with recurrent epithelium erosions were treated. The mean follow-up was two months. Significant pain reduction was observed $(p<0.001)$. Corneal thickness and visual acuity measurements presented with no significant difference. Conclusion: The potential use of cross-linking in the treatment of bullous keratopathy pain was proved to be likely. A longer follow-up to confirm whether these results can be reproduced in the long term is necessary.

Keywords: Cornea; Corneal topography; Cross-linking reagents; Corneal transplantation; Pain; Endothelium, corneal; Photosensitizing agents/therapeutic use; Riboflavin/therapeutic use; Ultraviolet rays; Visual acuity

\section{REFERÊNCIAS}

1. Georgiadis NS, Ziakas NG, Boboridis KG, Terzidou C, Mikropoulos DG. Cryopreserved amniotic membrane transplantation for the management of symptomatic bullous keratopathy. Clin Experiment Ophtalmol. 2008;36(2):130-5.

2. Haraguchi DK, Gomes JA, Sato EH, Novo NF. Uso de transplante de membrana amniótica no tratamento da ceratopatia bolhosa. Arq Bras Oftalmol. 2003; 66(1):121-4

3. Castellano AG, Bardal AM, Lago C, Moreira LB, Moreira H. Transplante de membrana amniótica na ceratopatia bolhosa. Arq Bras Oftalmol. 2004;67(6): 855-8.

4. Cormier G, Brunette I, Boisjoly HM, Le François M, Shi ZH, Guertin MC. Anterior stromal punctures for bullous keratopathy. Arch Ophthalmol. 1996; 114(6):654-8. 
5. Foulks GN. Treatment of recurrent corneal erosion and corneal edema with topical osmotic colloidal solution. Ophthalmology. 1981;88(8):801-3.

6. Luxenberg MN, Green K. Reduction of corneal edema with topical hypertonic agents. Am J Ophthalmol. 1971;71(4):847-53.

7. Plotnik RD, Mannis MJ, Schwab IR. Therapeutic contact lenses. Int Ophthalmol Clin. 1991;31(2):35-52.

8. Spoerl E, Huhle M, Seiler T. Induction of cross-links in corneal tissue. Exp Eye Res. 1998;66(1):97-103.

9. Spoerl E, Seiler T. Techniques for stiffening the cornea. J Refract Surg. 1999; 15(6):711-13

10. Spoerl E, Mrochen M, Sliney D, Trokel S, Seiler T. Safety of UVA-riboflavin cross-linking of the cornea. Cornea. 2007;26(4):385-9.

11. Wollensak G, Redl B. Gel electrophoretic analysis of corneal collagen after photodynamic cross-linking treatment. Cornea. 2008;27(3):353-6.

12. Wollensak G, Spoerl E, Seiler T. Riboflavin/ultraviolet-A-induced collagen crosslinking for the treatment of keratoconus. Am J Ophthalmol. 2003;135(5): 620-7.

13. Schnitzler E, Spoerl E, Seiler T. [Irradiation of cornea with ultraviolet light and riboflavin administration as a new treatment for erosive corneal process, preliminary results in four patients]. Klin Monatsbl Augenheilkd. 2000;217(3); 190-3. German.

14. Carvalho DS, Kowacs PA. Avaliação da intensidade de dor. Migrâneas e Cefaléias. 2006;9(4):164-8.

15. Conover WJ. Practical nonparametric statistics. $2^{\text {nd }}$ ed. New York. John Wiley \& Sons; 1980.

16. Krachmer JH, Mannis JM, Holland EJ. Cornea: fundaments diagnosis and management. $2^{\text {nd }}$ ed. Philadelphia: Elsevier Mosby; 2006.

17. Ehlers N, Hjortdal J. Riboflavin-ultraviolet light induced cross-linking in endothelial decompensation. Acta Ophthalmol. 2008:86(5)549-51.

18. Mazzotta C, Traversi C, Baiocchi S, Sergio P, Caporossi T, Caporossi A. Conservative treatment of keratoconus by riboflavin-UVA-induced cross-linking of corneal collagen: quantitative investigation of corneal epithelium and subepithelial nerve plexus regeneration by in vivo HRT II system confocal microscopy in humans. Eur J Ophthalmol. 2006:16(4):530-5.

19. Wollensak G, Aurich H, Pham DT, Wirbelauer C. Hydration behavior of porcine cornea crosslinked with ribloflavin and ultraviolet A. J Cataract Refract Surg. 2007;33(3):516-21. Comment in: J Cataract Refract Surg. 2007;33(9):503; J Cataract Refract Surg. 2008;34(6):879-80. 